\title{
Dark Sector Physics with Belle II
}

\section{Torben Ferber ${ }^{* \dagger}$}

University of British Columbia, Vancouver, British Columbia, Canada V6T 1 Z1

E-mail: ferber@physics.ubc.ca

The next-generation B-factory experiment Belle II at the upgraded KEKB accelerator, SuperKEKB, will start physics data taking in 2018. It is an asymmetric $e^{+} e^{-}$collider that will operate with $40 \times$ the instantaneous luminosity of KEKB/Belle and aims to collect $50 \times$ more data in total.

Belle II offers the possibility to search for a large variety of Dark Sector particles in the GeV mass range complementary to LHC and direct detection experiments. The searches at Belle II will profit both from the very large dataset, and from specifically designed triggers for the early running of Belle II.

EPS-HEP 2017, European Physical Society conference on High Energy Physics 5-12 July 2017

Venice, Italy

${ }^{*}$ Speaker.

${ }^{\dagger}$ on behalf of the Belle II collaboration 


\section{Searches For Dark Photons}

A possible extension of the SM are new U(1) gauge bosons, Dark Photons $A^{\prime}$, that couple via kinetic mixing to the SM photon $\gamma[1]$. The mixing strength can be expressed via the parameter $\varepsilon$ which can be understood as suppression factor relative to the electron charge $e$. At Belle II [2] a Dark Photon $A^{\prime}$ could be produced in the initial state radiation (ISR) accompanied reaction $e^{+} e^{-} \rightarrow A^{\prime} \gamma_{I S R}$ where the cross section is proportional to $\varepsilon^{2} \alpha^{2} / E_{C M}^{2}$ and $\alpha$ is the electromagnetic coupling.

If the $A^{\prime}$ is the lightest dark sector particle, it will decay into SM particles with branching ratios as a virtual photon of mass $M_{A^{\prime}}$. Several experiments have published results of $A^{\prime}$ searches and even more results are to come in the next years. The results of the BaBar experiment [3] for the decays of an $A^{\prime}$ in the $e^{+} e^{-}$and $\mu^{+} \mu^{-}$final states have been used to extract expected sensitivities for the Belle II experiment. The BaBar analysis is based on of a search for a narrow peak in the dilepton mass spectrum on top of a large - and apart from the proximity of narrow resonances smooth - QED SM background. Taking into account the better invariant mass resolution $(\sim$ factor 2$)$ of Belle II due to the larger drift chamber radius that improves the possible signal peak resolution and a better trigger efficiency for both muons $(\sim$ factor 1.1$)$ and electrons $(\sim$ factor 2$)$, the projected sensitivities for different values of integrated luminosity are shown in Fig. 1.

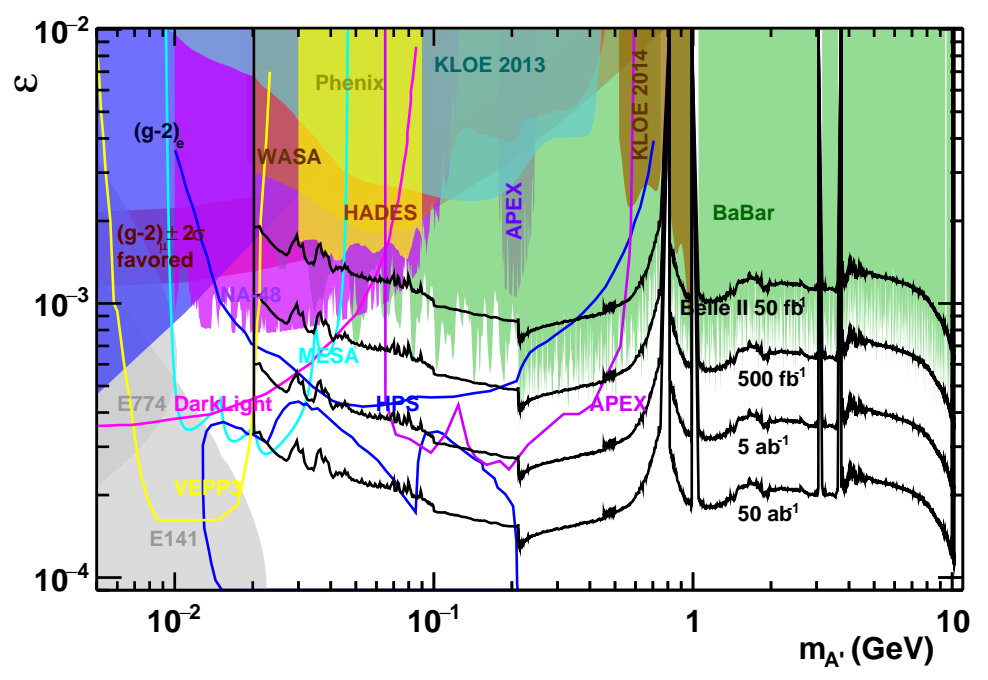

Figure 1: Existing exclusion regions (90\% CL) on the dark photon mixing parameter $\varepsilon$ and mass $M_{A^{\prime}}$ (solid regions) for $A^{\prime} \rightarrow \ell \ell$, with projected limits for Belle II and other future experiments (lines) (figure taken from [4]).

If the $A^{\prime}$ is not the lightest dark sector particle, it will dominantly decay into light Dark Matter (DM) $\chi$ via $A^{\prime} \rightarrow \chi \bar{\chi}$. Since the interaction probability of DM with the detector is negligible, the experimental signature of such a decay will be a mono-energetic ISR photon $\gamma_{I S R}$ with energy $E_{\gamma}=\left(E_{C M}^{2}-M_{A^{\prime}}^{2}\right) /\left(2 E_{C M}\right)$. A full detector simulation of QED backgrounds and signal efficiencies of Belle II was performed in order to determine the sensitivity to Dark Photons decaying into light 
DM [4]. The backgrounds for this search have been found to be due to high cross section QED processes $e^{+} e^{-} \rightarrow e^{+} e^{-} \gamma(\gamma)$ and $e^{+} e^{-} \rightarrow \gamma \gamma(\gamma)$ where all but one photon are undetected. The irreducible background from $e^{+} e^{-} \rightarrow v \bar{v} \gamma$ is negligible.

This analysis requires the implementation of a single-photon trigger that was not available at the predecessor experiment Belle. Based on simulation including full beam backgrounds, we expect a trigger rate of about $9 \mathrm{kHz}$ for a trigger based on one calorimeter cluster with $E>1 \mathrm{GeV}$ and not other calorimeter cluster with $E>0.1 \mathrm{GeV}$ in the tracking detector polar angle acceptance $17^{\circ}<\theta<150^{\circ}$. The rate is largely due to radiative Bhabha events in which there really is only a single photon in the acceptance of the detector.

The projected Belle II sensitivity for an integrated luminosity of $20 \mathrm{fb}^{-1}$ is shown in Fig. 2. The full expected Belle II dataset of $50 \mathrm{ab}^{-1}$ can improve the expected sensitivity by almost another order of magnitude. The much better expected sensitivity compared to BaBar for small mass Dark Photons is mainly due to the more homogeneous calorimeter of Belle II.

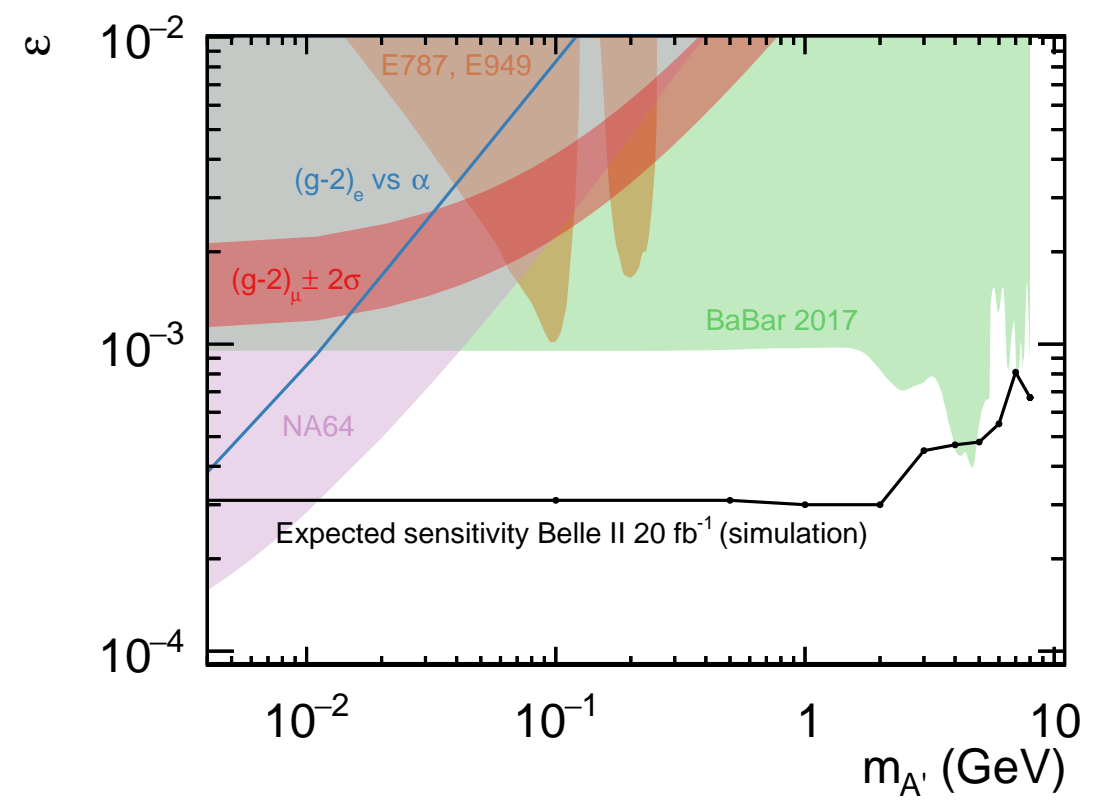

Figure 2: Projected upper limits on $\varepsilon$ for the process $e^{+} e^{-} \rightarrow \gamma A^{\prime}, A^{\prime} \rightarrow$ invisible, for a $20 \mathrm{fb}^{-1}$ data set (solid black curve) (figure taken from [4]).

\section{Searches For Axion-Like Particles}

Light pseudoscalar bosons that interact pre-dominantly with Standard Model gauge bosons, so-called axion-like particles (ALPs), occur frequently in extensions of the Standard Model. ALPs that coupled dominantly to photons with a coupling strength $g_{a g g}$ can be produced in two different ways at Belle II: In ALP-strahlung $\left(e^{+} e^{-} \rightarrow \gamma^{*} \rightarrow \gamma+a\right)$ and in photon fusion $\left(e^{+} e^{-} \rightarrow e^{+} e^{-}+a\right)$. 
Unless $m_{a}$ is close to $\sqrt{s}$, ALP production via photon fusion typically dominates over ALPstrahlung. However, the final state in photon fusion production features only two photons and missing momentum which will lead to very high QED background. The most promising search is hence for visibly and invisibly decaying ALPs, as well as long-lived ALPs from ALP-strahlung [5].

Invisibly decaying ALPs have the same experimental signature as Dark Photon decaying into $\mathrm{DM}$, but the angular distribution of the recoil photon is less peaked at small angles than the ISR photon in Dark Photon production. Using the the same event selection as for the Dark Photon search, the expected sensitivity for Belle II is shown in Fig. 3.

The experimental signature of visible ALP decays depends on their mass and coupling. For high ALP masses the small boost leads to a wide opening angle of the decay photons and a final state with three detectable photons. For smaller ALP masses, the higher boost leads to a decreased opening angle of the decay photons that cannot be separated in the calorimeter for $m_{a} \lesssim 200 \mathrm{MeV}$ in the offline reconstruction and $m_{a} \lesssim 400 \mathrm{MeV}$ in the hardware trigger. It is mandatory that events that appear as two photon events at hardware trigger level are not rejected or pre-scaled but passed to the offline reconstruction in order to maintain a high efficiency for low mass ALPs. The background is dominated by the QED process $e^{+} e^{-} \rightarrow \gamma \gamma \gamma$ with three photons in the final state. Additional small backgrounds for small ALP masses come from $e^{+} e^{-} \rightarrow \gamma \gamma$ with a third photon candidate coming from beam-induced backgrounds, and from $e^{+} e^{-} \rightarrow \gamma \gamma$ where one of the photon converts into an electron-positron pair outside of the tracking detectors. They can be reduced by vetoes on the photon time and the separation angle of the electron-positron pair in the magnetic field.

The sensitivity for long-lived ALPs can be determined from the sensitivity of ALP decays into DM, taking into account the reduced efficiency for ALPs decaying inside of Belle II.

The projected sensitivities to the coupling $g_{a g g}$ are summarized as a function of ALP mass $m_{a}$ in Fig. 3.

\section{Conclusions}

Belle II will be significantly more sensitive to low mass Dark Mediators and DM than existing experiments. Data taking will start in 2018 and dedicated triggers, a hermetic detector and improved offline reconstruction will give world leading sensitivity for invisible Dark Photon decays and searches for ALPs, even with the data taken in the first year only. Other Belle II searches in the Dark Sector will focus on displaced vertices of long-lived particles, light Higgs decays, and invisible $\Upsilon$ decays.

\section{References}

[1] B. Holdom, Phys.Lett. B166, 196 (1986). 

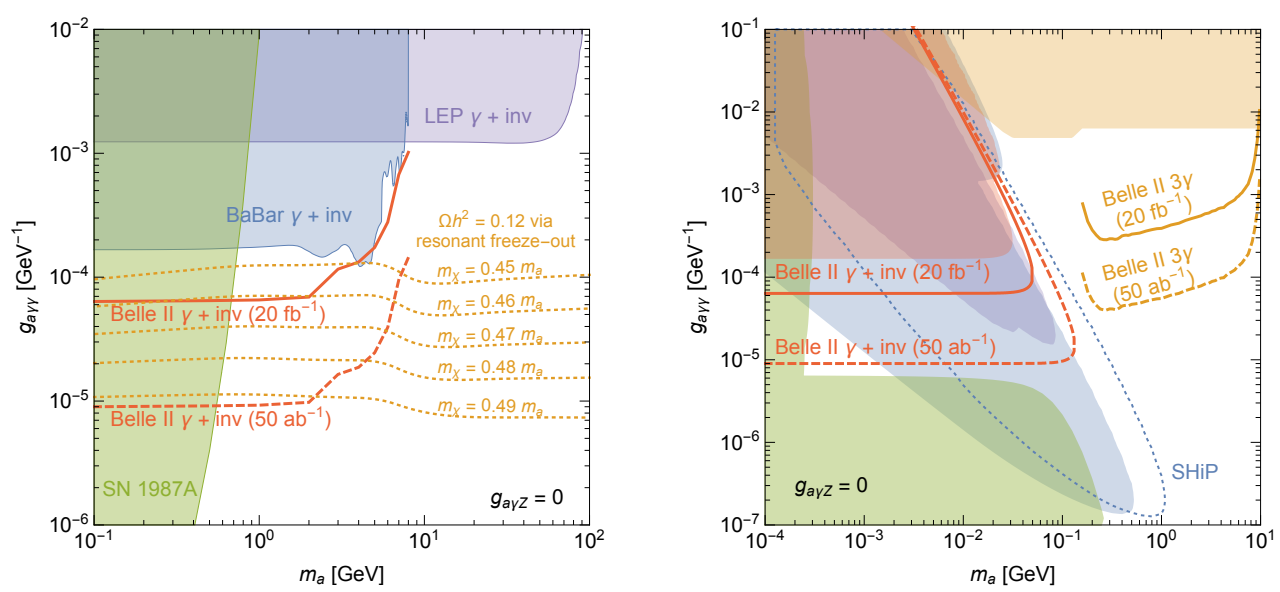

Figure 3: Projected Belle II sensitivity ( $90 \%$ CL) compared to existing constraints on ALPs decaying into DM compared to the parameter region where one can reproduce the observed DM relic abundance via resonant annihilation of DM into photons (left). Projected Belle II sensitivity (90\% CL) compared to existing constraints on ALPs with photon coupling, as well as the projected sensitivity from SHiP (right) (figures taken from [5]).

[2] T. Abe et al., Belle II Technical Design Report, KEK Report 2010-1, arXiv:1011.0352 (2010).

[3] J. P. Lees et al., Phys. Rev. Lett. 113, 201801 (2014).

[4] Belle II Collaboration and the B2TIP Theory Community, "The Belle II Physics Book", to be submitted to PTEP (2017).

[5] M. Dolan, T. Ferber, C. Hearty, F. Kahlhoefer and K. Schmidt-Hoberg, submitted to JHEP (2017), arXiv:1709.00009. 